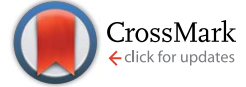

Cite this: RSC Adv., 2017, 7, 12017

\title{
Heme protein-mediated synthesis of PEDOT:PSS: enhancing conductivity by inhibiting heme degradation $\uparrow$
}

\begin{abstract}
J. J. Flores, ${ }^{a b c}$ C. K. Payne ${ }^{a b}$ and J. D. Morris ${ }^{\star a b c}$
Poly(3,4-ethylenedioxythiophene):poly(styrenesulfonate) (PEDOT:PSS) is a conducting polymer used in regenerative medicine, solar energy conversion, OLEDs, and biological sensing. PEDOT:PSS can be synthesized with a wide range of biomolecular oxidants including hemoglobin, catalase, horseradish peroxidase, soybean peroxidase, and laccase. Unfortunately heme proteins have been found to degrade during polymer synthesis, limiting their utility. We show that the peroxidase substrate, 2,2'-azino-bis(3ethylbenzothiazoline-6-sulphonic acid) (ABTS), inhibits heme degradation during hemoglobin-mediated synthesis of PEDOT:PSS, measured by fluorescence emission. Four-point probe measurements show that films of PEDOT:PSS are more conductive when synthesized in the presence of ABTS. Characterization of the resulting PEDOT:PSS films using visible and near IR spectroscopy shows that ABTS produces a bipolaron rich polymer, as expected if heme degradation is inhibited. Conductivity is further enhanced $\left(31 \mathrm{~S} \mathrm{~cm}^{-1}\right.$ ) when an iron chelator, EDTA, is used in combination with ABTS.
\end{abstract}

Received 20th January 2017

DOI: $10.1039 / \mathrm{c} 7 \mathrm{ra00887b}$

rsc.li/rsc-advances

iron in a heme complex and are combined with hydrogen peroxide as an oxidant. Examples include catalase, ${ }^{40}$ cytochrome $c{ }^{38}$ hemoglobin, ${ }^{41}$ horseradish peroxidase, ${ }^{37}$ and soybean peroxidase. $^{38,42,43}$ Non-heme biomolecules used for synthesis include laccase $^{\mathbf{4 4}}$ and transferrin. ${ }^{\mathbf{4 0}}$ As this list makes clear, enzymatic activity is not required for polymerization. ${ }^{40}$ The acidic conditions required for PEDOT:PSS synthesis ( $\mathrm{pH} 1-2)$ means most biomolecules will be denatured..$^{25}$ A notable exception is laccase, which can enzymatically polymerize PEDOT:PSS under more mild conditions ( $\mathrm{pH}$ 3.5). ${ }^{44}$ Additionally, soybean peroxidase can enzymatically polymerize PEDOT:PSS at a $\mathrm{pH}$ of 5 . In this case, however, terthiophene was added to the reaction as a radical initiator. $^{43}$ Biomolecular synthesis of PEDOT:PSS results in conductivities ranging from $1 \times 10^{-5} \mathrm{~S} \mathrm{~cm}^{-1}$ (catalase) to $2.8 \mathrm{~S}$ $\mathrm{cm}^{-1}$ (hemoglobin). ${ }^{\mathbf{4 0 , 4 1}} \mathrm{A}$ critical factor for heme proteins used in PEDOT:PSS synthesis is the relative concentration of hemebound iron compared to unbound, free, iron. ${ }^{41}$ Heme iron, combined with hydrogen peroxide, generates a highly conductive polymer dominated by bipolarons. ${ }^{45}$ While free iron, also combined with hydrogen peroxide, generates a weakly conductive polymer dominated by polarons. ${ }^{25,45}$ Iron chelators, such as ethylenediaminetetraacetic acid (EDTA), can be used to prevent unbound iron from participating in the synthesis. ${ }^{45}$ For this reason, the addition of EDTA during hemoglobin mediated PEDOT:PSS polymerizations leads to a significant conductivity enhancement from $2.8 \mathrm{~S} \mathrm{~cm}^{-1}$ to $19.5 \mathrm{~S} \mathrm{~cm}^{-1} .41$

The balance of free iron versus heme iron is largely determined by the reaction of hydrogen peroxide with heme iron. ${ }^{38}$ Biomolecular oxidants require hydrogen peroxide for synthesis, but hydrogen peroxide also causes the degradation of the heme 
group and the release of free iron. ${ }^{46-48}$ While EDTA inhibits free iron from synthesizing PEDOT:PSS, it does not prevent the degradation of the heme group. We hypothesized that the conductivity of hemoglobin synthesized PEDOT:PSS would be enhanced by inhibiting heme degradation with $2,2^{\prime}$-azino-bis(3ethylbenzothiazoline-6-sulfonic acid) (ABTS), a peroxidase substrate. ${ }^{46,47,49,50}$

In this work we show that polymerization of PEDOT:PSS in the presence of ABTS inhibits heme degradation and significantly enhances conductivity. When ABTS is combined with EDTA during synthesis, we observe further enhancement of polymer conductivity to $31 \mathrm{~S} \mathrm{~cm}^{-1}$. To our knowledge, this represents a record conductivity for undoped PEDOT:PSS. We then investigate the underlying mechanism of this enhancement. We use visible and near IR spectroscopy to characterize the resulting PEDOT:PSS films. We find that the suppression of heme degradation by ABTS leads to bipolaron-dominated PEDOT:PSS.

PEDOT:PSS was synthesized by combining hemoglobin $(4.8 \mu \mathrm{M}$ by heme concentration), EDOT (50 mM) and PSS (25 mM, by monomer concentration) in an $\mathrm{HCl}-\mathrm{KCl}$ buffer ( $\mathrm{pH}$ 1.15) while stirring. The reaction was initiated by the addition of $\mathrm{H}_{2} \mathrm{O}_{2}(20$ $\mathrm{mM}$ ). The reaction was allowed to proceed for three hours followed by dialysis. For full experimental details, please see the ESI. $\dagger$

The combination of hemoglobin with hydrogen peroxide results in the degradation of the heme group and the release of free iron. ${ }^{46,47}$ In the context of hemoglobin-mediated synthesis of PEDOT:PSS, this leads to a less conductive polymer dominated by polarons. ${ }^{34}$ The peroxidase substrate, ABTS, however, inhibits the degradation of hemoglobin at neutral $\mathrm{pHs}^{46,47,49,50}$ If heme degradation is inhibited under our reaction conditions $(\mathrm{pH}=$ 1.15), then the addition of ABTS during synthesis will enhance PEDOT:PSS conductivity. As a control, we synthesized hemoglobin without ABTS and used four point probe measurements to determine its conductivity $\left(0.81 \mathrm{~S} \mathrm{~cm}^{-1}\right.$, Fig. 1$)$.

Next we synthesized PEDOT:PSS in the presence of ABTS. We found that $0.4 \mathrm{mM}$ ABTS (Fig. S1, ESI $\dagger$ ) led to the most promising polymer with a threefold conductivity enhancement to 2.6 $\mathrm{S} \mathrm{cm}^{-1}$ (Fig. 1). This finding is consistent with our hypothesis that ABTS inhibits heme degradation during PEDOT:PSS synthesis. This is expected to increase conductivity for two reasons. First, in the absence of ABTS, the degradation of hemoglobin by hydrogen peroxide will result in a continual decrease in oxidant concentration, lowering yield and decreasing the final PEDOT:PSS ratio. The ratio of conductive PEDOT to insulating PSS critically determines the final conductivity of the resulting polymer films. ${ }^{25,51}$ Second, the degradation of hemoglobin results in the release of free iron, as discussed above. Any PEDOT oxidized by free iron and hydrogen peroxide is expected to have low conductivity $\left(\sim 10^{-7} \mathrm{~S} \mathrm{~cm}^{-1}\right){ }^{41}$

The conductivity of the polymer synthesized with hemoglobin and ABTS is relatively high for undoped PEDOT:PSS, but it remains lower than the previously reported combination of hemoglobin with EDTA $\left(19.5 \mathrm{~S} \mathrm{~cm}^{-1}\right) .{ }^{41}$ The present synthesis was done under slightly different conditions including a lower hemoglobin concentration, a lower hydrogen peroxide concentration, and a shorter reaction duration. Both the lower concentration of hemoglobin and the shorter reaction time will lower the

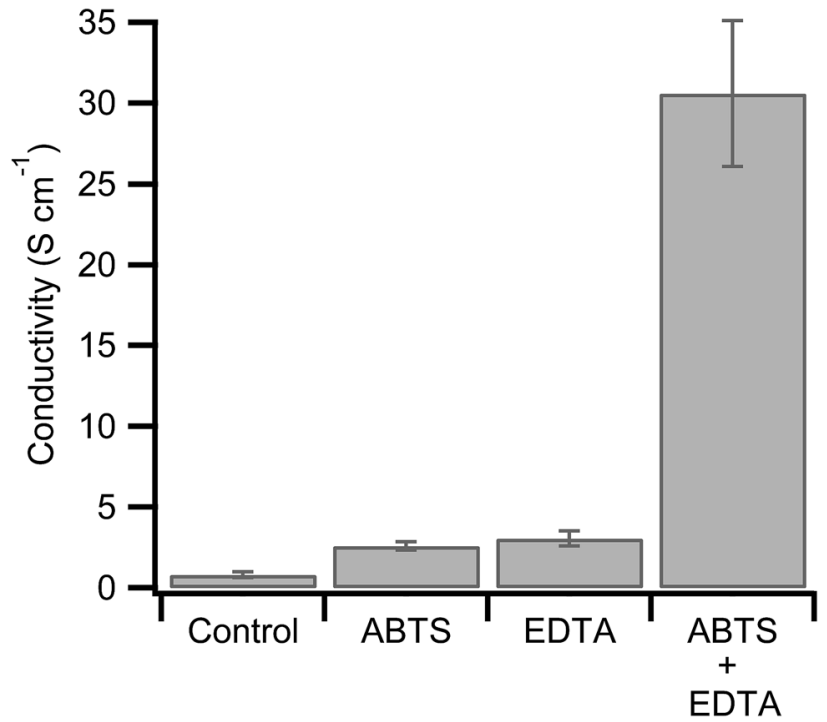

Fig. 1 Conductivity of PEDOT:PSS polymerized by hemoglobin alone (control) or combined with ABTS, EDTA, or both ABTS and EDTA, as determined by four-point probe. The film thicknesses of each polymer were $2.40 \pm 0.17 \mu \mathrm{m}, 1.83 \pm 0.17 \mu \mathrm{m}, 4.53 \pm 0.89 \mu \mathrm{m}$ and $2.83 \pm 0.24$ $\mu \mathrm{m}$, respectively. The error bars represent the standard error of a minimum of 10 measurements across 3 films.

final yield of PEDOT. Since the PSS template is already polymerized (MW $=70000 \mathrm{kDa}$ ), this will lower the PEDOT:PSS ratio. As described above, this is expected to lower conductivity. ${ }^{25,51}$ For comparison with the previous result, we synthesized PEDOT:PSS with hemoglobin and EDTA under our reaction conditions. The resulting polymer has a conductivity of $3.06 \mathrm{~S} \mathrm{~cm}^{-1}$, similar to the polymerization in the presence of ABTS (Fig. 1).

While ABTS may prevent the release of free iron from the heme group, it does not prevent trace iron from participating in the polymerization. For this reason we polymerized PEDOT:PSS with hemoglobin in the presence of both ABTS and EDTA in the same polymerization. PEDOT:PSS synthesized under these conditions shows a conductivity of $31 \mathrm{~S} \mathrm{~cm}^{-1}$. This represents a $50 \%$ increase over the highest conductivity previously obtained from biomolecular synthesis and, to our knowledge, represents a record conductivity for undoped PEDOT:PSS. ${ }^{41}$

Besides the previously mentioned EDTA and hemoglobin polymerization, the next highest conductivity reported for undoped PEDOT:PSS is $10 \mathrm{~S} \mathrm{~cm}^{-1}$. ${ }^{52}$ For this synthesis, Qi, et al. formed an emulsion of EDOT in PSS. This allowed more EDOT to be dissolved in an aqueous solution and ultimately led to a higher PEDOT:PSS ratio. Investigations by Aleshin, et al. reported a conductivity of $20 \mathrm{~S} \mathrm{~cm}^{-1} .^{53}$ In this case, however, commercial PEDOT:PSS was cast from low concentration HCl. It is well known that $\mathrm{HCl}$, as well as other acids, are effective postpolymerization treatments to enhance the conductivity of PEDOT:PSS ${ }^{54,55}$ Indeed films of PEDOT:PSS doped by acid can achieve conductivities as high as $4000 \mathrm{~S} \mathrm{~cm}^{-1} .{ }^{27,56}$ While films treated with organic solvents reach $1100 \mathrm{~S} \mathrm{~cm}^{-1} .^{28}$ For a comprehensive discussion of PEDOT:PSS conductivity enhancements see the review by Shi, et al. ${ }^{26}$ Importantly, ABTS and EDTA do not alter PEDOT:PSS after polymerization, rather 
they optimize the oxidant used for polymerization. We are directly producing highly conductive PEDOT:PSS in a one-step, aqueous reaction.

Previous work has shown that PEDOT:PSS oxidized by heme iron results in a higher bipolaron to polaron ratio compared to PEDOT:PSS oxidized with free iron..$^{\mathbf{3 8 4 1 4 5}}$ If ABTS limits heme degradation, we should see an increase in the bipolaron to polaron ratio. Polarons in PEDOT:PSS produce an absorption at $\sim 800 \mathrm{~nm}$ while bipolarons in PEDOT:PSS absorb at $\sim 3000 \mathrm{~nm}^{.25,45,57}$ To compare the bipolaron to polaron ratio, we characterized each polymer with visible and near IR spectroscopy from $350 \mathrm{~nm}$ to $2500 \mathrm{~nm}$ in a single spectra (Fig. 2a). These measurements were taken on polymer films cast on glass, as the water in aqueous solutions of PEDOT:PSS absorbs strongly in the IR. ${ }^{58}$ The measured film thicknesses of each polymer blend varied from $2 \mu \mathrm{m}$ (hemoglobin alone) to $4 \mu \mathrm{m}$ (hemoglobin and ABTS). For this reason, the absorbance at $800 \mathrm{~nm}$ was used to normalize each spectrum. This normalization allows us to compare the bipolaron to polaron ratio, but not absolute absorption. The control, synthesized using only hemoglobin, shows the lowest absorption at $2500 \mathrm{~nm}$ relative to the absorption at $800 \mathrm{~nm}$ (Fig. 2b). This indicates that the bipolaron to polaron ratio is the lowest for the control. The absorbance at $2500 \mathrm{~nm}$, and hence the bipolaron to polar ratio, increases for PEDOT:PSS polymerized in the presence of ABTS or EDTA. The combination of ABTS and EDTA leads to the highest bipolaron to polaron absorption ratio. Since heme iron is expected to produce a bipolaron rich polymer, these observations are consistent with our hypothesis that ABTS inhibits heme degradation. ${ }^{45}$ To ensure this change is due to an interaction between heme and ABTS, we polymerized PEDOT:PSS with $\mathrm{FeCl}_{3}$ in the presence of ABTS. No significant change in the visible and near IR spectra was observed (Fig. S2, ESI $†$ ).

The impact of EDTA on the near IR absorbance is similar to ABTS, but for a different reason. EDTA removes free iron, while ABTS preserves more heme iron. Both effects lead to a bipolaron rich polymer. A detailed discussion of the impact of EDTA on the biomolecular synthesis of PEDOT:PSS has been published previously. ${ }^{41}$ Additionally, we find that higher bipolaron absorption in the near IR correlates with greater polymer conductivity. This suggests that the increased conductivity may arise in part due to an increase in oxidation state of the polymer.

It is well known that ABTS inhibits heme degradation at physiological pHs. ${ }^{46,47,49,50}$ To confirm this inhibition also occurs under our reaction conditions of $\mathrm{pH} 1.15$, we directly monitored the impact of ABTS on the reaction of hemoglobin with hydrogen peroxide. The absorption of ABTS prevents monitoring heme degradation by UV-Vis spectroscopy due to spectral overlap (Fig. S3, ESI $\dagger$ ). Fortunately, the degradation products from the reaction of hydrogen peroxide with hemoglobin are fluorescent. $^{46,47}$ Although the chemical identity of these products is unclear, fluorescence has been used previously to observe the impact of ABTS on heme degradation. ${ }^{\mathbf{4 6 , 4 7}}$ Hemoglobin was incubated with hydrogen peroxide in $\mathrm{HCl}-\mathrm{KCl}(\mathrm{pH} 1.15)$ for 60 minutes and then an emission scan was collected (excite: $320 \mathrm{~nm}$, Fig. 3a). The fluorescence intensity of the heme degradation products is observed as a broad peak centered at $445 \mathrm{~nm}$. In the absence of hemoglobin or $\mathrm{H}_{2} \mathrm{O}_{2}$, no peak is observed at (a)
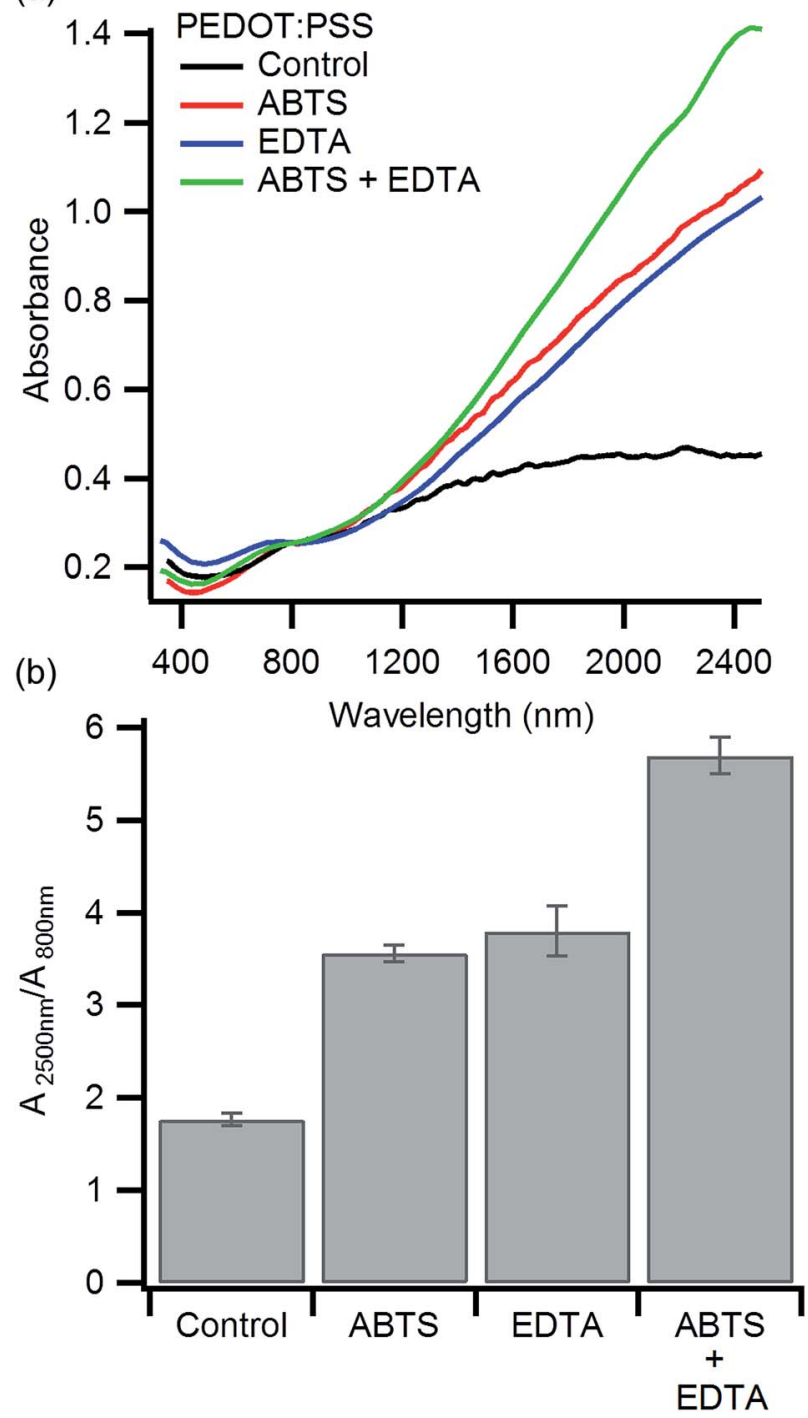

Fig. 2 (a) Representative visible and near IR spectra of PEDOT:PSS polymerized by hemoglobin alone (control, black), in the presence of ABTS (red), EDTA (blue), and both ABTS and EDTA (green). (b) The ratio of absorbance at $2500 \mathrm{~nm}$ to $800 \mathrm{~nm}$ of PEDOT:PSS polymerized by hemoglobin alone (control), in the presence of ABTS, EDTA, and both ABTS and EDTA. Error bars represent the standard error of three separate polymerizations under each condition.

$445 \mathrm{~nm}$ (Fig. S4, ESI $\dagger$ ). Previous investigations, at neutral pH, show an emission peak at a slightly higher wavelength of $465 \mathrm{~nm} .{ }^{\mathbf{4 6 , 4 7}}$ This shift is likely due to the change in $\mathrm{pH}$ which can alter the wavelength of fluorescence emission. ${ }^{59}$ The fluorescence intensity at $445 \mathrm{~nm}$ is significantly reduced in the presence of ABTS indicating heme degradation is suppressed.

We then monitored the increase in fluorescence emission overtime at $445 \mathrm{~nm}$ (Fig. 3b). The fluorescence increases gradually overtime both with and without ABTS. The data imply heme degradation is reduced by ABTS, but not inhibited entirely. The initial $(t=0)$ high intensity of fluorescence in the presence of ABTS is from a fluorescence peak at $470 \mathrm{~nm}$ due to ABTS itself. ${ }^{60}$ This peak is no longer significant after 10 minutes of exposure to hydrogen peroxide (Fig. S5 $†$ ). 

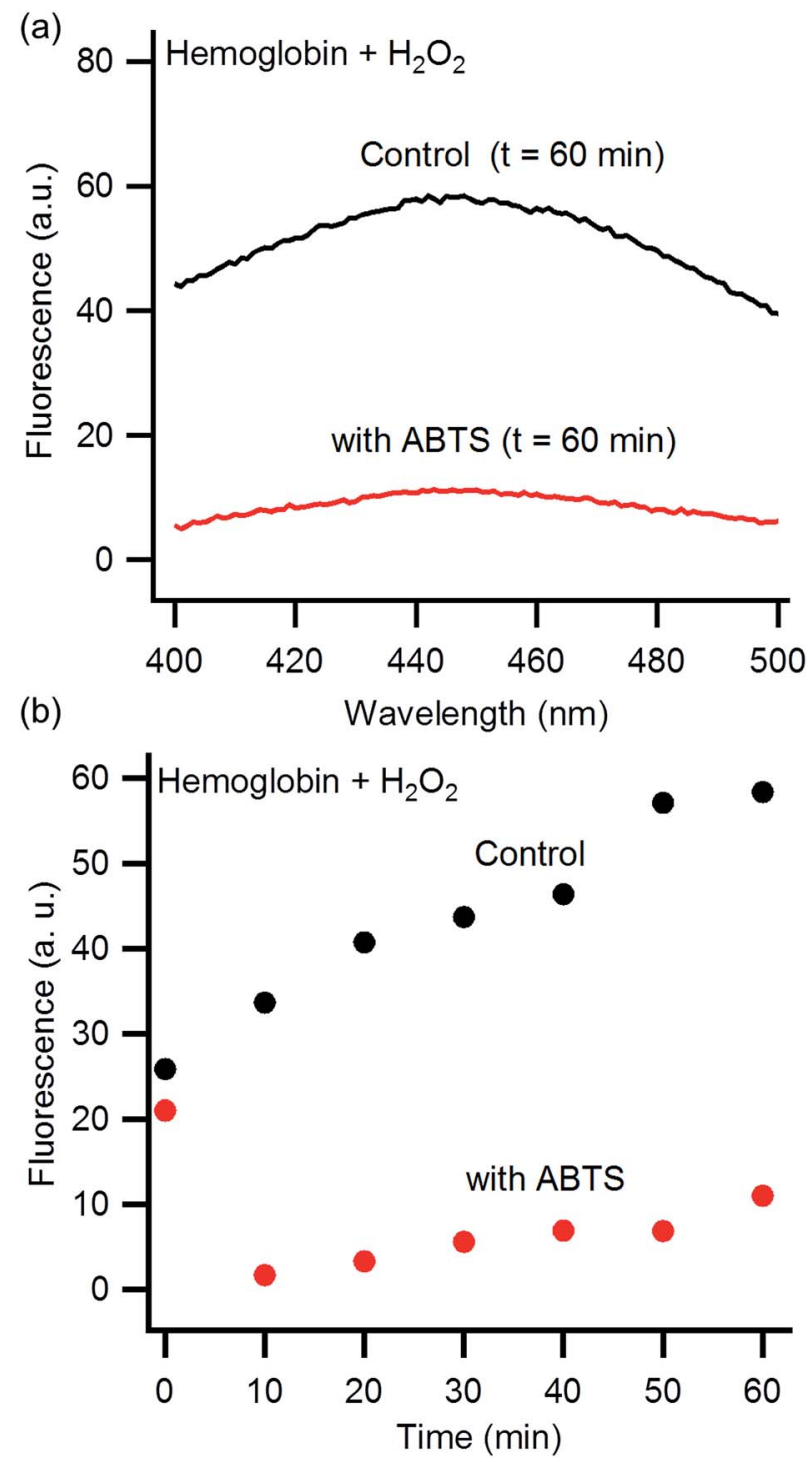

Fig. 3 Fluorescence emission of hemoglobin combined with hydrogen peroxide at $\mathrm{pH} 1.15$ (excite: $325 \mathrm{~nm}$ ). (a) Representative fluorescence emission spectra of hemoglobin 60 minutes after exposure to hydrogen peroxide in the presence (red) and absence (black) of ABTS. (b) Kinetic plot of fluorescence emission intensity in the presence (red) and absence (black) of ABTS (emit: $445 \mathrm{~nm}$ ). The initial $(t=0)$ ) high intensity of fluorescence in the presence of ABTS is from a fluorescence peak at $470 \mathrm{~nm}$ due to ABTS itself. ${ }^{60}$ This peak is no longer significant after 10 minutes of exposure to hydrogen peroxide (Fig. S5 $\dagger$ ).

To understand the mechanism by which ABTS inhibits heme degradation, it is important to consider the interaction of hydrogen peroxide and acid denatured hemoglobin. Under our reaction conditions, hemoglobin possesses ferrous iron (Fe(II)). ${ }^{41,61-63}$ When hydrogen peroxide reacts with ferrous hemoglobin, it can generate ferrylhemoglobin $(\mathrm{Fe}(\mathrm{Iv})=\mathrm{O}) .{ }^{64}$ The subsequent reaction of ferrylhemoglobin with another equivalent of hydrogen peroxide, produces superoxide and methemoglobin (Fe(III)). ${ }^{46}$ Superoxide then degrades the heme group and releases free iron. ABTS inhibits this degradation by reducing ferrylhemoglobin to methemoglobin. ${ }^{46}$ Methemoglobin can still react with another equivalent of hydrogen peroxide, but in this case, it produces the radical oxoferrylhemoglobin $\left({ }^{\circ} \mathrm{Fe}(\mathrm{rv})=\mathrm{O}\right)$. Hydrogen peroxide reacting oxoferrylhemoglobin still produces methemoglobin but with oxygen, instead of superoxide. In this way, ABTS protects hemoglobin from degradation. For more details on this process see the work by Nagababu, et al. ${ }^{46}$

\section{Conclusions}

We have demonstrated that ABTS can be used in hemoglobinmediated PEDOT:PSS synthesis to enhance polymer conductivity (Fig. 1). When EDTA is also added during synthesis, the conductivity is further enhanced to $31 \mathrm{~S} \mathrm{~cm}^{-1}$. These increases in conductivity are correlated with an increased bipolaron to polaron absorption ratio in the near IR (Fig. 2). This suggests the conductivity is enhanced due to an increase in PEDOT:PSS oxidation state. Finally, we confirmed that ABTS inhibits heme degradation under our acidic reaction conditions (Fig. 3). These experiments confirm our hypothesis that inhibiting heme degradation during PEDOT:PSS synthesis increases polymer conductivity. This work highlights the importance of understanding the underlying mechanistic details of biomolecular polymer synthesis to synthesize high conductivity PEDOT:PSS in a one-step, aqueous reaction.

\section{Acknowledgements}

This work was supported by a Vasser Woolley Faculty Fellowship to CKP, an NSF REU Grant (CHE-1560335) to Georgia Tech, and by the School of Science and Technology at Georgia Gwinnnett College through its STEC 4500 research program.

\section{Notes and references}

1 B. L. Groenendaal, F. Jonas, D. Freitag, H. Pielartzik and J. R. Reynolds, Adv. Mater., 2000, 12, 481-494.

2 S. Kirchmeyer and K. Reuter, J. Mater. Chem., 2005, 15, 20772088.

3 K. Sun, S. Zhang, P. Li, Y. Xia, X. Zhang, D. Du, F. Isikgor and J. Ouyang, J. Mater. Sci.: Mater. Electron., 2015, 26, 44384462.

4 M. Dietrich, J. Heinze, G. Heywang and F. Jonas, J. Electroanal. Chem., 1994, 369, 87-92.

5 G. Heywang and F. Jonas, Adv. Mater., 1992, 4, 116-118.

6 X. Cui and D. C. Martin, Sens. Actuators, B, 2003, 89, 92-102.

7 K. A. Ludwig, J. D. Uram, J. Yang, D. C. Martin and D. R. Kipke, J. Neural. Eng., 2006, 3, 59-70.

8 T. F. Otero and J. G. Martinez, J. Mater. Chem. B, 2016, 4, 2069-2085.

9 F. Jonas, W. Krafft and B. Muys, Macromol. Symp., 1995, 100, 169-173.

10 W. H. Kim, A. J. Makinen, N. Nikolov, R. Shashidhar, H. Kim and Z. H. Kafafi, Appl. Phys. Lett., 2002, 80, 3844-3846.

11 C. Zhong, C. Duan, F. Huang, H. Wu and Y. Cao, Chem. Mater., 2010, 23, 326-340.

12 C. A. Zuniga, S. Barlow and S. R. Marder, Chem. Mater., 2011, 23, 658-681. 
13 F. Chen, Q. Lin, H. Wang, L. Wang, F. Zhang, Z. Du, H. Shen and L. S. Li, Nanoscale Res. Lett., 2016, 11, 376.

14 W. J. Hong, Y. X. Xu, G. W. Lu, C. Li and G. Q. Shi, Electrochem. Commun., 2008, 10, 1555-1558.

15 G. Li, V. Shrotriya, J. Huang, Y. Yao, T. Moriarty, K. Emery and Y. Yang, Nat. Mater., 2005, 4, 864-868.

16 F. L. Zhang, A. Gadisa, O. Inganäs, M. Svensson and M. R. Andersson, Appl. Phys. Lett., 2004, 84, 3906-3908.

17 Ö. Yagci, S. S. Yesilkaya, S. A. Yüksel, F. Ongül, N. M. Varal, M. Kus, S. Günes and O. Icelli, Synth. Met., 2016, 212, 12-18.

18 F. Ongul, S. A. Yuksel, M. Kazici, S. Bozar, A. Gunbatti and S. Gunes, Polym. Adv. Technol., 2015, DOI: 10.1002/pat.3677.

19 E. P. Tomlinson, M. E. Hay and B. W. Boudouris, Macromolecules, 2014, 47, 6145-6158.

20 K. C. Donavan, J. A. Arter, R. Pilolli, N. Cioffi, G. A. Weiss and R. M. Penner, Anal. Chem., 2011, 83, 2420-2424.

21 J. A. Arter, D. K. Taggart, T. M. McIntire, R. M. Penner and G. A. Weiss, Nano Lett., 2010, 10, 4858-4862.

22 N. K. Guimard, N. Gomez and C. E. Schmidt, Prog. Polym. Sci., 2007, 32, 876-921.

23 S. M. Richardson-Burns, J. L. Hendricks and D. C. Martin, J. Neural. Eng., 2007, 4, L6-L13.

24 S. M. Richardson-Burns, J. L. Hendricks, B. Foster, L. K. Povlich, D. H. Kim and D. C. Martin, Biomaterials, 2007, 28, 1539-1552.

25 A. Elschner, S. Kirchmeyer, W. Lovenich, U. Merker and K. Reuter, PEDOT: Principles and Applications of an Intrinsically Conductive Polymer, CRC Press, Boca Raton, Fl, 2010.

26 H. Shi, C. Liu, Q. Jiang and J. Xu, Adv. Electron. Mater., 2015, 1, 150017.

27 C. Yeon, S. J. Yun, J. Kim and J. W. Lim, Adv. Electron. Mater., 2015, 1, 1500121.

28 K. Lim, S. Jung, S. Lee, J. Heo, J. Park, J.-W. Kang, Y.-C. Kang and D.-G. Kim, Org. Electron., 2014, 15, 1849-1855.

29 S. Kobayashi, H. Uyama and S. Kimura, Chem. Rev., 2001, 101, 3793-3818.

30 S. Kobayashi, J. Polym. Sci., Part A: Polym. Chem., 1999, 37, 3041-3056.

31 B. Ryan, K. Akshay, R. Sethumadhavan, N. Subhalakshmi, K. Jayant, A. S. Lynne, F. B. Ferdinando and N. Ramaswamy, in Green Polymer Chemistry: Biocatalysis and Biomaterials, American Chemical Society, 2010, vol. 1043, ch. 23, pp. 315-341.

32 W. Liu, J. Kumar, S. Tripathy, K. J. Senecal and L. Samuelson, J. Am. Chem. Soc., 1999, 121, 71-78.

33 K. S. Alva, J. Kumar, K. A. Marx and S. K. Tripathy, Macromolecules, 1997, 30, 4024-4029.

34 W. Liu, S. Bian, L. Li, L. Samuelson, J. Kumar and S. Tripathy, Chem. Mater., 2000, 12, 1577-1584.

35 J. S. Dordick, M. A. Marletta and A. M. Klibanov, Biotechnol. Bioeng., 1987, 30, 31-36.

36 H. Uyama, H. Kurioka, I. Kaneko and S. Kobayashi, Chem. Lett., 1994, 23, 423-426.

37 V. Rumbau, J. A. Pomposo, A. Eleta, J. Rodriguez, H. Grande, D. Mecerreyes and E. Ochoteco, Biomacromolecules, 2007, 8, 315-317.
38 J. D. Morris, K. M. Wong, C. D. Penaherrera and C. K. Payne, Biomater. Sci., 2016, 4, 331-337.

39 R. A. Gross, A. Kumar and B. Kalra, Chem. Rev., 2001, 101, 2097-2124.

40 S. M. Hira and C. K. Payne, Synth. Met., 2013, 176, 104-107. 41 J. D. Morris, D. Khanal, J. A. Richey and C. K. Payne, Biomater. Sci., 2015, 3, 442-445.

42 A. Tewari, A. Kokil, S. Ravichandran, S. Nagarajan, R. Bouldin, L. A. Samuelson, R. Nagarajan and J. Kumar, Macromol. Chem. Phys., 2010, 211, 1610-1617.

43 S. Nagarajan, J. Kumar, F. F. Bruno, L. A. Samuelson and R. Nagarajan, Macromolecules, 2008, 41, 3049-3052.

44 G. Shumakovich, G. Otrokhov, I. Vasil'eva, D. Pankratov, O. Morozova and A. Yaropolov, J. Mol. Catal. B: Enzym., 2012, 81, 66-68.

45 J. D. Morris and C. K. Payne, Org. Electron., 2014, 15, 17071710.

46 E. Nagababu and J. M. Rifkind, Biochemistry, 2000, 39, 12503-12511.

47 E. Nagababu and J. M. Rifkind, Biochem. Biophys. Res. Commun., 1998, 247, 592-596.

48 J. M. C. Gutteridge, FEBS Lett., 1986, 201, 291-295.

49 R. E. Childs and W. G. Bardsley, Biochem. J., 1975, 145, 93103.

50 M. B. Arnao, M. Acosta, J. A. del Rio and F. García-Cánovas, Biochim. Biophys. Acta, 1990, 1038, 85-89.

51 A. M. Nardes, M. Kemerink, R. A. J. Janssen, J. A. M. Bastiaansen, N. M. M. Kiggen, B. M. W. Langeveld, A. J. J. M. van Breemen and M. M. de Kok, Adv. Mater., 2007, 19, 1196-1200.

52 Z. Qi and P. G. Pickup, Chem. Commun., 1998, 2299-2300, DOI: $10.1039 /$ A805322G.

53 A. N. Aleshin, S. R. Williams and A. J. Heeger, Synth. Met., 1998, 94, 173-177.

54 Y. Xia and J. Ouyang, ACS Appl. Mater. Interfaces, 2010, 2, 474-483.

55 Y. Xia, K. Sun and J. Ouyang, Adv. Mater., 2012, 24, 24362440.

56 N. Kim, S. Kee, S. H. Lee, B. H. Lee, Y. H. Kahng, Y.-R. Jo, B.-J. Kim and K. Lee, Adv. Mater., 2014, 26, 2268-2272.

57 F. L. E. Jakobsson, X. Crispin, L. Lindell, A. Kanciurzewska, M. Fahlman, W. R. Salaneck and M. Berggren, Chem. Phys. Lett., 2006, 433, 110-114.

58 R. F. Goddu and D. A. Delker, Anal. Chem., 1960, 32, 140-141. 59 J. R. Lakowicz, Principles of fluorescence spectroscopy, Springer Science \& Business Media, 2013.

60 C. Lee and J. Yoon, J. Photochem. Photobiol., A, 2008, 197, 232-238.

61 M. F. Perutz, J. K. M. Sanders, D. H. Chenery, R. W. Noble, R. R. Pennelly, L. W. M. Fung, C. Ho, I. Giannini, D. Poerschke and H. Winkler, Biochemistry, 1978, 17, 36403652 .

62 E. A. Rachmilewitz, J. Peisach and W. E. Blumberg, J. Biol. Chem., 1971, 246, 3356-3366.

63 B. L. Horecker, J. Biol. Chem., 1943, 148, 173-183.

64 C. Giulivi and K. J. A. Davies, in Methods Enzymol., Academic Press, 1994, vol. 231, pp. 490-496. 